\title{
Drug Complexation and Formulation of a Buccal Tablet
}

\author{
Rajashree Hirlekar* and Vilasrao Kadam
}

\author{
Department of Pharmaceutics, Bharati Vidyapeeth's College of Pharmacy, Sec-8, CBD, Belapur, Navi \\ Mumbai 400614 (M.S.), India
}

\begin{abstract}
In the present work use of Beta-Cyclodextrin for inclusion of Carvedilol, an antihypertensive drug with poor solubility and high first pass metabolism was studied, in order to improve its dissolution rate. The complex prepared by kneading method was characterized by Differential Scanning Calorimetry, Fourier Transformation Infra Red Spectroscopy and X-Ray Diffractometry along with the plain drug and physical mixture. The drug so complexed was incorporated into a buccal dosage form to by pass first pass metabolism to improve its bioavailability. The buccal tablets containing complex showed reasonable mucoadhesive strength. The dissolution and permeability across pig buccal mucosa were improved in case of tablets containing the complex as compared to plain drug tablet.
\end{abstract}

Key words: Carvedilol, Beta-Cyclodextrin, Complexation, Dissolution, Characterization, Permeation.

\section{Introduction}

Carvedilol (CAR) is a non selective $\beta$ adrenergic antagonist widely used to treat hypertension and angina pectoris (Moser and Frishman 1998). It is sparingly soluble in water (Dunn et al. 1997) and shows high first pass metabolism (Mollendroff and Neugebauer 1987). The low drug bioavailability is attributed to these two factors. The first part of the present work aims at improving the water solubility of the drug by forming the inclusion complex with Beta Cyclodextrin ( $\beta$ CD). CDs are water soluble cyclic oligosaccharides with a cone shaped structure. Due to their particular conformation they are able to include a guest molecule inside their hydrophobic cavity forming a noncovalently bonded complex, resulting in its improved solubility. Solubility of various drugs has been improved by CD complexation (Miyake et al.1999; Longxiao and Suyan. 2006; Perdomo- Lopez et al. 2002; Badawy et al. 1996; Ammar et al.2006). Buccal route has been successfully utilized for administration of drugs undergoing high first pass metabolism and degradation in the harsh gastrointestinal environment (Miyazaki et al. 1995). Buccal drug absorption can be promptly terminated in case of toxicity by removing the dosage form from the buccal cavity. CAR exhibits high first pass metabolism of $80 \%$ (Mollendroff and Neugebauer 1987). Hence its bioavailability can be increased by administration by buccal route. The second part of the present work involves formulation of a buccal tablet incorporating the CAR- $\beta C D$ complex.

\footnotetext{
* Corresponding author: E-mail: rshirlekar@rediffmail.com
}

\section{Materials and Methods}

CAR was kindly supplied by Sun Pharmaceuticals, India. $\beta C D$ (M. W. = 1135) was gifted by Signet chemical Corporation. Carbopol $974 \mathrm{P}$ was received as a gift sample from Noveon. These chemicals were used as received without further purification. All other reagents were of analytical reagent grade purity. Double distilled water was used throughout the study.

\section{Preparation and characterization of CAR- $\beta C D$ binary systems}

For preparation of binary systems, CAR and $\beta C D$ were used in 1:1 stoichiometric ratio. Physical mixtures were prepared by mixing the drug and $\beta C D$ and passing through $100 \#$ sieve. For the kneaded product, the mixture was triturated for $15 \mathrm{~min}$ in a mortar followed by addition of minimum amount of $66 \%$ alcohol. The mixture was ground thoroughly for 45 min to obtain paste which was dried at $60^{\circ} \mathrm{C}$ overnight. The resultant powder was ground, passed through 100\# sieve and stored in a dessicator. CAR- $\beta C D$ binary systems were characterized by Differential Scanning Calorimetry (DSC) on a Mettler Tolledo DSC 822. Samples of drug, $\beta C D$ and binary mixtures containing drug were placed in sealed aluminum pans and heated at $10^{\circ} \mathrm{C} / \mathrm{min}$ in the range of $30-200^{\circ} \mathrm{C}$, using an empty sealed pan as a reference. Dry nitrogen was used as 
purge gas. The powder X-ray diffraction patterns of CAR, $\beta C D, P M$ and inclusion complex were recorded using Phillips P Analytical X'Pert PRO powder X-Ray diffractometer (Netherlands) using Ni-filtered, $\mathrm{CuK} \alpha$ radiation, a voltage of $40 \mathrm{kV}$ and a current of $30 \mathrm{~mA}$. The scanning rate employed was $1^{\circ}$ per min and samples were analyzed between 2 angles of over 5-45 . Infra-Red spectra were obtained using Jasco-700 FTIR Spectrophotometer using $\mathrm{KBr}$ discs. The instrument was operated under dry air purge and the scans were collected at scanning speed of $2 \mathrm{~mm} / \mathrm{sec}$ with resolution of $4 \mathrm{~cm}^{-1}$ over the region of $4000-400 \mathrm{~cm}^{-1}$.

\section{Preparation of buccal tablets}

For preparation of buccal tablets, CAR binary system equivalent to $6.25 \mathrm{mg}$ of CAR, $63.24 \mathrm{mg}$ of lactose DCL, $7.5 \mathrm{mg}$ of carbopol 974 P, 15 mg sodium carboxy methyl cellulose, $1 \mathrm{mg}$ of magnesium stearate and $2 \mathrm{mg}$ of talc previously screened through 100\# sieve were mixed and compressed on a single punch machine using $9 \mathrm{~mm}$ circular flat beveled punch. The backing membrane of ethyl cellulose was compressed over it in order to get unidirectional release of the drug. For comparison purpose tablets containing only CAR were also prepared.

\section{In vitro dissolution studies}

Dissolution of CAR from the CAR- $\beta$ CD complex and buccal tablets containing CAR- $\beta C D$ complex equivalent to 10 $\mathrm{mg}$ and $6.25 \mathrm{mg}$ of CAR respectively were evaluated in buffer pH 6.8 using USP XXIII dissolution apparatus type-II (6 stations, VDA-6DR, Veego Scientific, India) at $37 \pm$ $0.5^{\circ} \mathrm{C}$ stirring at $50 \mathrm{rpm}$. For tablets $900 \mathrm{ml}$ and for complex $1000 \mathrm{ml}$ of dissolution medium was used. The samples were withdrawn at predetermined time intervals and analyzed spectrophotometrically at $285 \mathrm{~nm}$.

\section{Porcine buccal mucosa preparation}

Porcine buccal tissue from domestic pigs was obtained from local slaughterhouse. The mucosal membrane was excised by removing connective and adipose tissue and was equilibrated at $37 \pm 0.1^{\circ} \mathrm{C}$ for $30 \mathrm{~min}$ in phosphate buffer $\mathrm{pH}$ 7.4.

\section{Ex Vivo drug permeation studies}

The buccal epithelium was carefully mounted in between the two compartments of Modified Franz Diffusion cell. Buccal tablets containing plain drug and complexed drug were tested. The tablets were stuck to the mucosa in the donor side.
Receiver medium was a mixture of alcohol, propylene glycol, phosphate buffer pH (7.4) in a ratio of 40:15:45 maintained at $37 \pm 0.1^{\circ} \mathrm{C}$ under gentle stirring. From the receiver compartment, $2 \mathrm{ml}$ aliquot was collected at predetermined time intervals and replaced by an equal volume of alcohol, propylene glycol, phosphate buffer solution. Analysis of samples was performed using a Jasco 2000 HPLC system equipped with a pump-PU 2080, UV / Vis detector (Jasco UV 2075) and a reverse phase column HIQSIL C18 (250 X $4.6 \mathrm{~mm}, 5 \mu \mathrm{m})$ at ambient temperature. The mobile phase was a mixture acetonitrile and phosphate buffer $(0.05 \mathrm{M}$ $\mathrm{KH}_{2} \mathrm{PO}_{4}$ at $\left.\mathrm{pH} 4.5\right)(60: 40, \mathrm{v} / \mathrm{v})$ run at $0.8 \mathrm{ml} / \mathrm{min}$.

\section{Measurement of mucoadhesive force}

The mucoadhesive force was checked by using Modified Balance Test (Gupta et al. 1992). Porcine buccal mucosa was tied with its mucosal side out onto the lower teflon cylinder. The tablet was stuck to the upper teflon cylinder using cyanoacrylate adhesive and lowered onto the mucosa under constant weight of $5 \mathrm{~g}$ for a total contact time of $1 \mathrm{~min}$. Mucoadhesive strength was assessed in terms of weight required to detach the tablet from the membrane.

\section{Results and Discussion}

The DSC profiles of pure components (CAR and $\beta C D)$ and binary systems in melting range of the drug and carriers dehydration are shown in Fig. 1. The thermogram of CAR was typical of a highly crystalline compound, characterized by a sharp endothermic peak at $118^{\circ} \mathrm{C}$ which corresponded to its melting (Miro et al. 2006). The DSC thermogram of $\beta C D$ showed a broad endothermic effect around in the range of $100-120^{\circ} \mathrm{C}$, associated to crystal water loss from $\beta C D$ (Hassan et al. 1990). The endothermic peaks of drug and $\beta C D$ were retained in PM showing no interaction between them. In case of $\mathrm{KN}$ systems the endothermic peak of drug disappeared and new one appeared at $93^{\circ} \mathrm{C}$, similar to observation made for haloperidol (Loukas et al. 1997). The XRD pattern for CAR (Fig. 2) indicated characteristic peaks which were retained in the case of PM. However, the XRD pattern of KN sample was devoid of crystalline drug peaks which were similar to observation made in the case of budesonideHPßCD complex (Bandi et al. 2004). FTIR spectrum of CAR (Fig. 3) indicated aromatic secondary C-N vibrations at 1253, C-C multiple bond stretching at 1502 and C-H stretching of aromatic ring at $2922 \mathrm{~cm}^{-1}$ which remained 


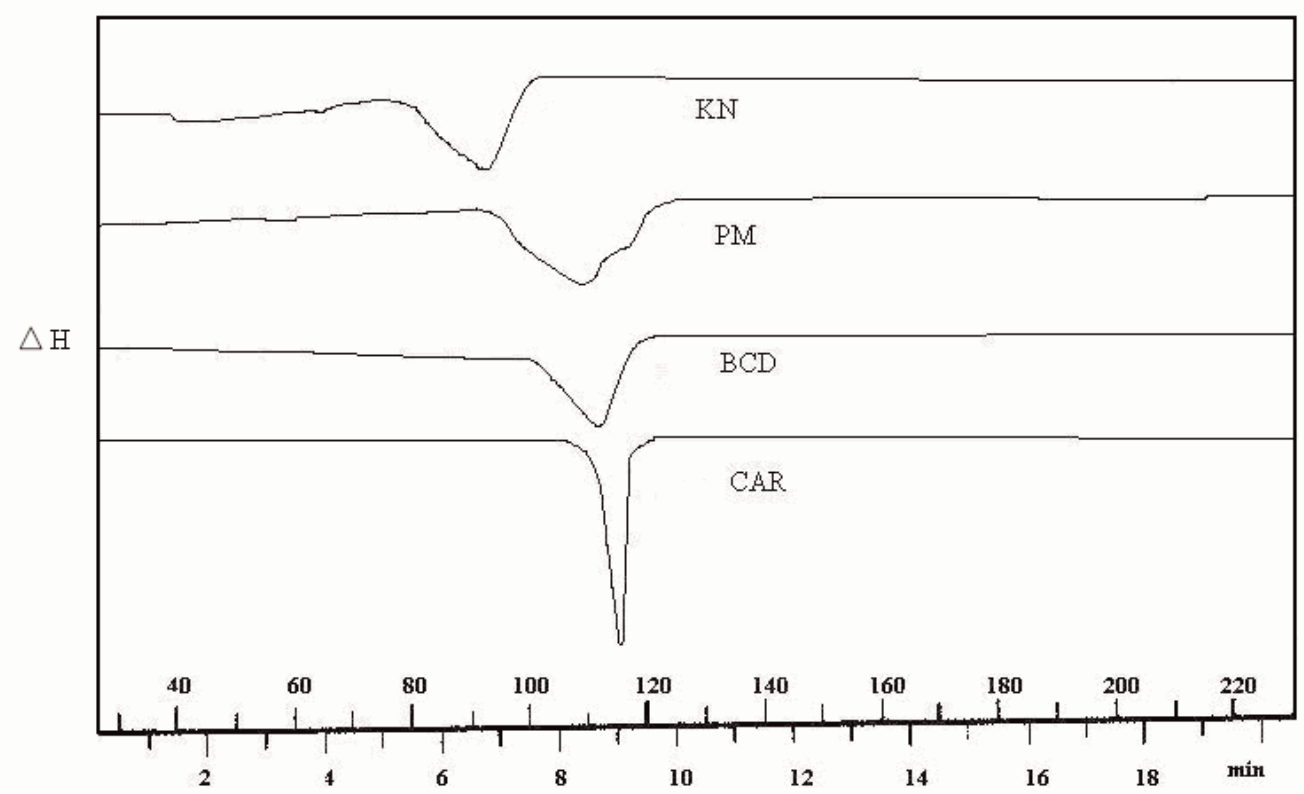

Fig. 1. DSC Thermograms of CAR- $\beta C D$ system: Carvedilol (CAR), $\beta$-cyclodextrin (BCD), Physical mixture (PM) and Kneaded (KN) system

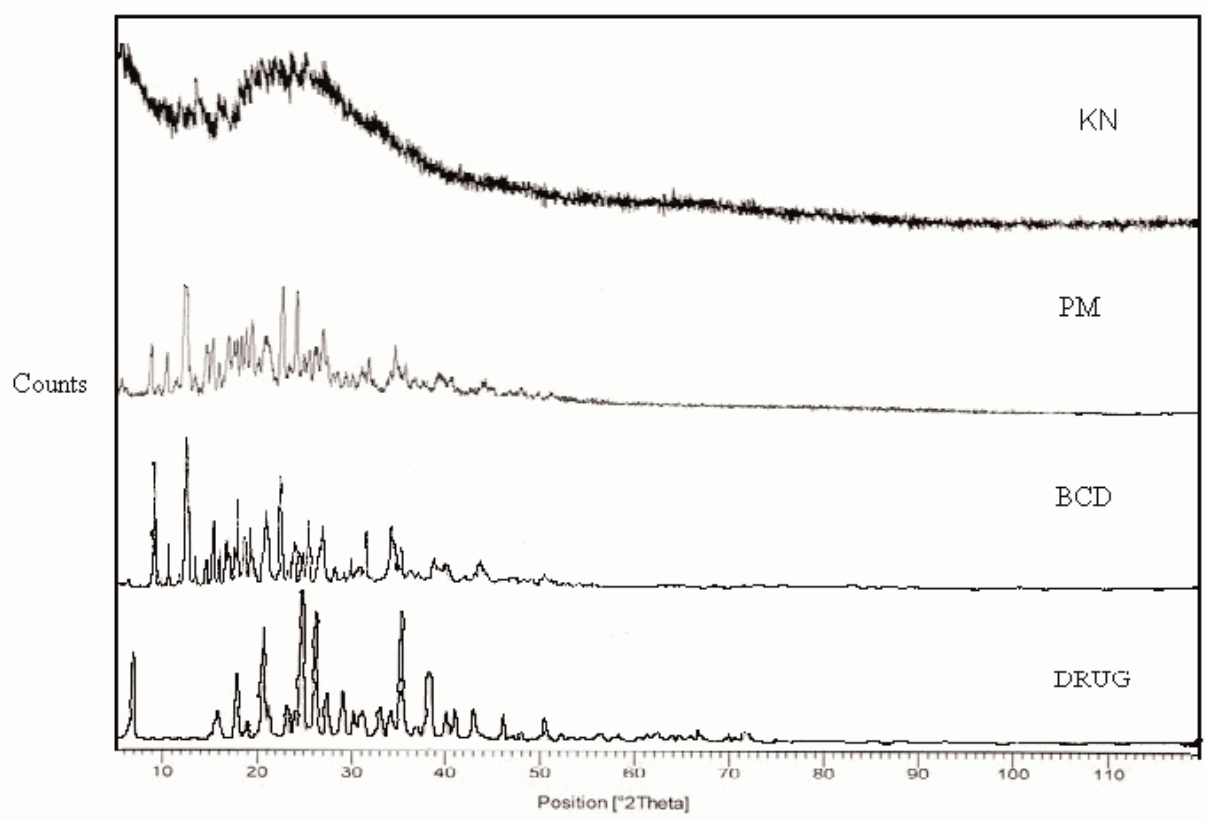

Fig. 2. X-Ray Diffractograms of CAR- $\beta C D$ system: Carvedilol (CAR), $\beta$-cyclodextrin (BCD), Physical mixture (PM) and Kneaded (KN) system

unchanged in the physical mixture. CAR- $\beta C D$ KN sample showed disappearance of the above characteristic peaks, suggesting possibility of entrapment of CAR into the host cavity during inclusion complexation.
The dissolution rate profiles of CAR and its binary systems are reported in Fig. 4. Dissolution of CAR was incomplete even after 3 hrs. The KN product gave complete release at the end of 120 mins. Improvement in dissolution rate of 


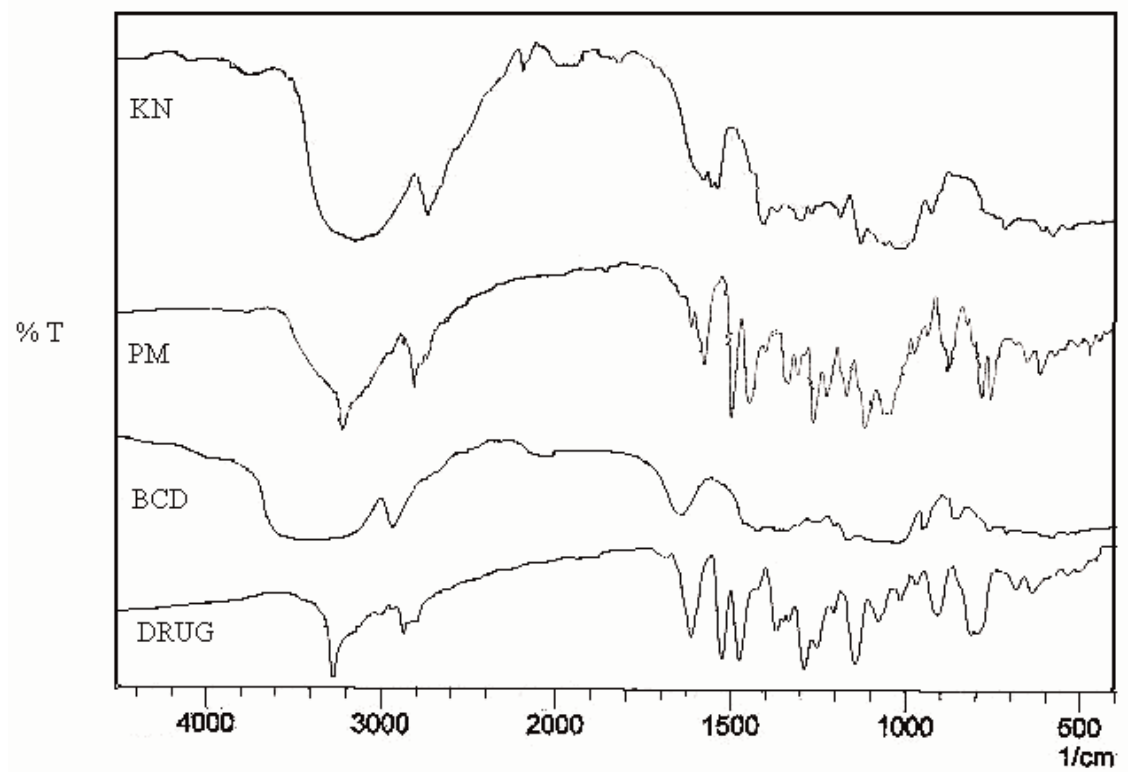

Fig. 3. FTIR spectra of CAR- $\beta C D$ system: Carvedilol (CAR), $\beta$-cyclodextrin (BCD), Physical mixture (PM) and Kneaded (KN) system

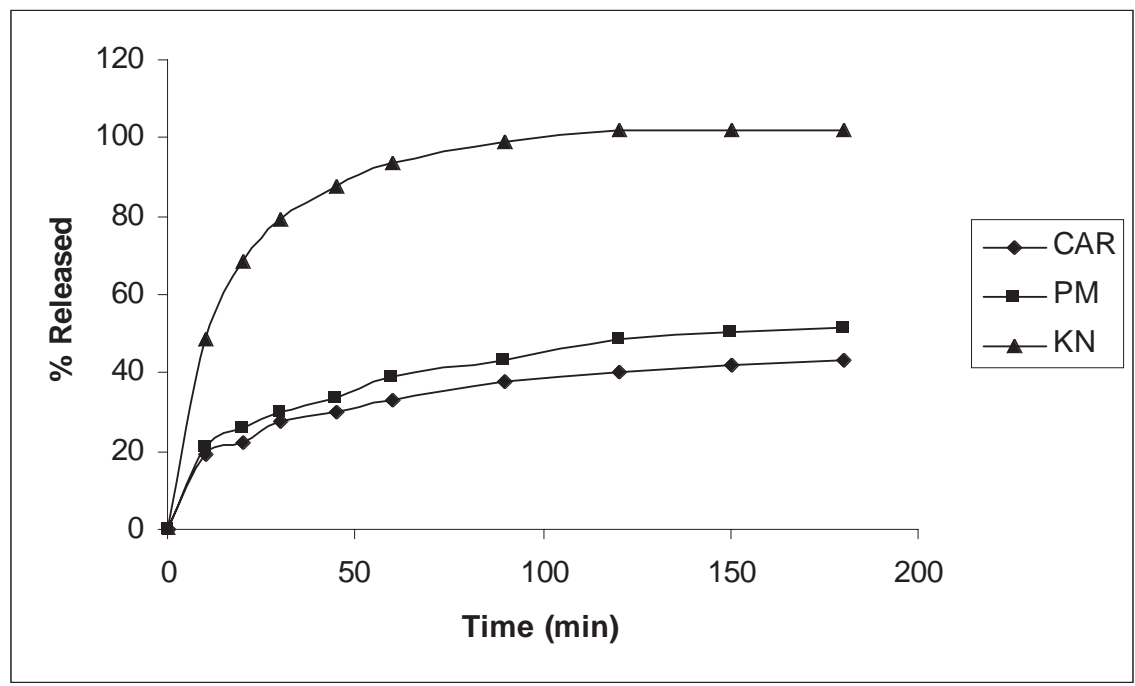

Fig. 4. Dissolution profile of CAR- $\beta C D$ system in phosphate buffer pH 6.8. Carvedilol (CAR), Physical mixture (PM) and Kneaded (KN) system

Meloxicam (Abdoh et al. 2007), Imatinib (Beni et al. 2007), Bupivacaine (Morales et al. 2007) and Sidenafil (Al Omari et al. 2006) has been reported after complexation with cyclodextrins. Results of in vitro dissolution studies of tablets are shown in Fig 5. Complete release of CAR was obtained from tablets containing complexed drug as compared to $41.22 \%$ from tablets containing plain drug at the end of 3.5 hrs. There have been controversial reports regard ing the effect of $\beta C D$ on dissolution of drug from tablet formulations. It has been demonstrated that dissolution rate of Diltiazem from the tablets was decreased by addition of $\beta C D$ (Horiuchi et al. 1990). Increased dissolution by inclusion complexation with $\beta C D$ has been observed for Nifedipine (Chowdary and Kamalakara 2003). Enhanced dissolution by $\beta C D$ tends to be observed with water insoluble drugs, presumably since dissolution of water insoluble drugs in the 


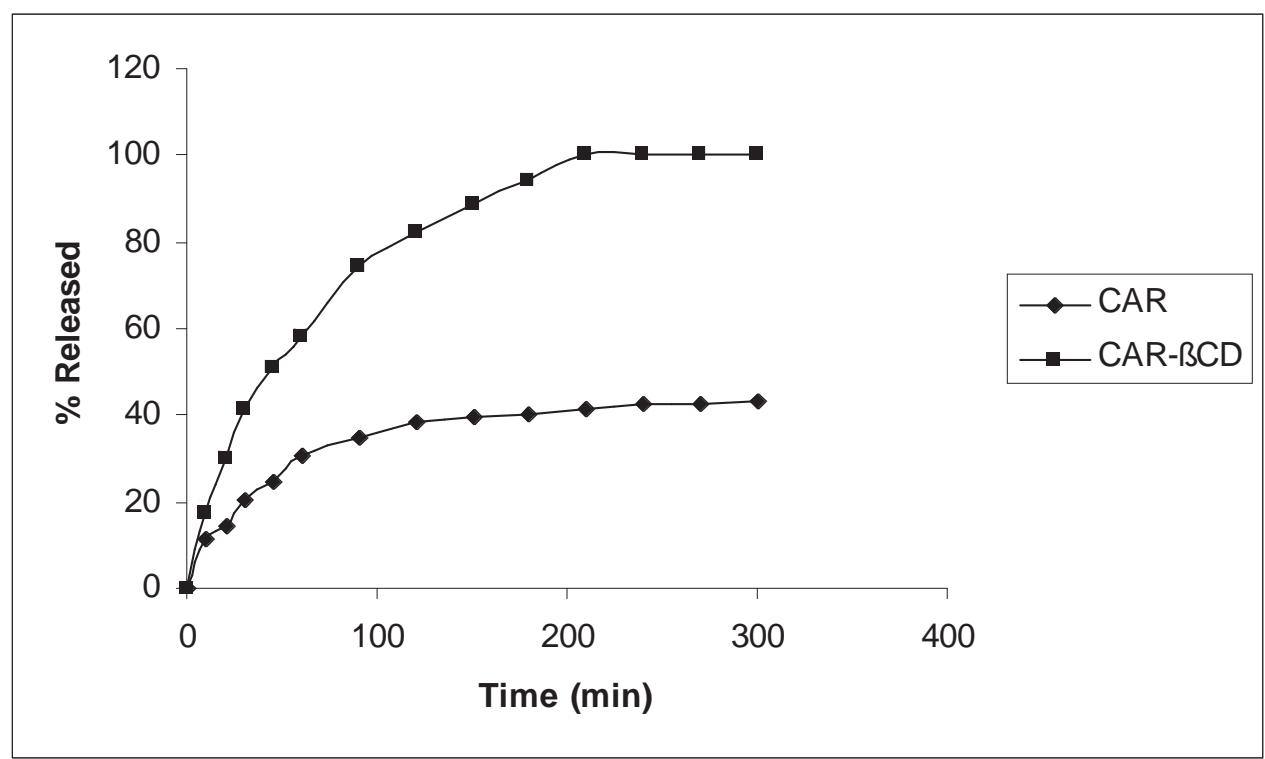

Fig. 5. Dissolution profile of CAR tablets in phosphate buffer pH 6.8. Carvedilol (CAR), Kneaded (KN) system

crystal form would be slower than that for inclusion complexes. CAR being poorly soluble drug, it's dissolution from tablets containing complex was improved as compared to the one containing plain drug.

Amount of drug permeated across the porcine buccal mucosa was $5.7 \mathrm{mg}$ and $2.51 \mathrm{mg}$ from tablets containing complexed drug and plain drug respectively. The results stressed the effectiveness of $\beta C D$ in improving the permeability of CAR across the mucous membrane. It has been reported that permeation of CAR was improved in the case tablets containing CAR-HP $\beta C D$ complex as compared to plain drug (Cappello et al. 2006). Usefulness of CP as buccal mucoadhesive polymer has been reported (Nafee et al. 2004). Hence CP 974P was selected in the present formulation. Both the buccoadhesive tablets containing complexed drug and plain drug showed satisfactory mucoadhesive strength of $10 \mathrm{~g}$.

\section{Conclusion}

Results of characterization studies indicated that CAR could form inclusion complex with $\beta$-CD and improved its dissolution rate. The buccal tablets containing CAR- $\beta C D$ complex showed better drug dissolution and permeability across buccal mucosa as compared to those containing plain drug. The tables had satisfactory mucoadhesive strength. Thus $\beta$ $\mathrm{CD}$ can be used satisfactorily to improve dissolution of CAR and use of buccal tablets can possibly improve the bioavailability of CAR by avoiding FPM.

\section{References}

Abdoh A., El-Barghouthi M., Zughul M., Davies J. and Badwah A. (2007) Changes in the conformational structure, microscopic and macroscopic pKa's of meloxicam on complexation with natural and modified cyclodextrins. Pharmazie. 62: 55-59.

Al Omari M., Zughul M., Davies J. and Badwah A. (2006) Sidenafil/cyclodextrin complexation: stability constants, thermodynamics and guest-host interactions probed by $1 \mathrm{H}$ NMR and molecular modeling studies. $J$. Pharm. Biomed. Anal. 41: 857-865.

Ammar H. O., Salama H. A., Ghorab M. and Mahmoud A. A. (2006) Formulation and biological evaluation of glimepiride-cyclodextrin-polymer system. Int. J. Pharm. 309: 129-138.

Badawy S. I. F., Ghorab M. M. and Adeyeye C. M (1996) Bioavailability of danazol-hydroxypropyl- $\beta$-cyclodextrin complex by different routes of administration. Int. J. Pharm. 145: 137-143.

Bandi N., Wei W., Roberts C. B., Kotra L. P. and Kompella U. B. (2004) Preperation of budesonide- and indomethacin-hydroxypropyl- $\beta$-cyclodextrin (HPBCD) complexes using a single-step, organic-solvent-free supercritical fluid process. Eur. J. Pharm. Sci. 23: 159-168. 
Beni S., Szakacs Z., Csernak O., Bareza L. and Noszal B. (2007) Cyclodextrin and imatinib complexation: binding mode and charge dependent stabilities. Eur. J. Pharm. Sci. 30: 167-174.

Cappello B., Rosa G. D., Giannini L., La Rotonda M. I., Mensitieri G., Miro A., Quaglia F. and Russo R. (2006) Cyclodextrin-containing poly (ethyleneoxide) tablets for the delivery of poorly soluble drugs: Potential as buccal delivery system. Int. J. Pharm. 319 (1-2): 6370.

Chowdary K. P. R. and Kamalakara R. G. (2003) Controlled release of nifedipine from mucoadhesive tablets of its inclusion complexes with $\beta$-cyclodextrin. Pharmazie. 58: $721-724$.

Dunn C. J., Lea A. P. and Wagstaff A. J. (1997) Carvedilol: a reappraisal of its pharmacological properties and therapeutic use in cardiovascular disorders. Drugs. 54: 161-185.

Gupta A., Garg S. and Khar R. K. (1992) Measurement of bioadhesive strength of mucoadhesive buccal tablets: design of an in-vitro assembly. Indian Drugs. 30(4): 152-155.

Hassan M., Suleiman M. and Najib N. (1990) Improvement of the in vitro dissolution characteristics of famotidine by inclusion in $\beta$-cyclodextrin. Int. J. Pharm. 58: 19- 24.

Horiuchi Y., Hirayama F. and Uekama K. (1990) slow release characteristics of diltiazem from ethylated $\beta$ cyclodextrin complexes. J. Pharm. Sci. 79: 128-132.

Longxiao L. and Suyan Z. (2006) Preparation and characterization of inclusion complexes of prazosin hydrochloride with $\beta$-cyclodextrin and hydroxypropyl- $\beta$ cyclodextrin. J. Pharm. Biomed. Anal. 40: 122-127.

Loukas Y. L., Vraka V. and Gregoriadis G. (1997) Novel nonacidic formulations of haloperidol complexed with $\beta$ cyclodextrin derivatives. J. Pharm. Biomed. Anal.16: 263-268.
Miro A., Quaglia F., Giannini L., Cappello B. and Maria I. L. R. (2006) Drug/ Cyclodextrin Solid Systems in the Design of Hydrophilic Matrices: A Strategy to Modulate Drug Delivery Rate. Cur. Drug. Deliv. 3: 373-378.

Miyake K., Irie T., Arima H., Hirayam F., Uekama K., Hirano M. and Okamoto Y. (1999) Characterization of itraconazole-2-hydroxypropyl- $\beta$-cyclodextrin inclusion complex in aqueous propylene glycol solution. Int. J. Pharm. 179: 237-245.

Miyazaki S., Nakayama M., Oda M., Takada M. and Attwood D. (1995) Drug release from oral mucosal adhesive tablets of chitosan and sodium alginate. Int. J. Pharm. 118: 257-263.

Mollendroff E. V. and Neugebauer R. K. (1987) Pharmacokin- etics and bioavailability of carvedilol, a vasodialating beta blocker. Eur. J. Clin. Pharmcol. 33: 511-513.

Morales C., Abrami P., Paula E., Braga A. and Fraceto L. (2007) Study of the interaction between S (-) bupivacaine and 2-hydroxypropyl- $\beta$-cyclodextrin. Int. J. Pharm. 331: 99-106.

Moser, M. Frishman, W.H. (1998) Results of thera with carvedilol, a $\beta$-blocker vasodilator with antioxidant properties, in hypertensive patients. Am. J. Hypertens. 11: $15 S-22 S$.

Nafee N. A., Ismail F. A., Boraie N. A. and Mortada L. M. (2004) Mucoadhesive delivery systems. I. Evaluation of mucoadhesive polymers for buccal tablet formulation. Drug. Dev. Ind. Pharm. 30 (9): 985-993.

Perdomo- Lopez I., Rodriguez-Perez A. I., Yzquierdo-Peiro J. M., White A., Estrada E. G., Villa T. G. and TorresLabandeira J. J. (2002) Effect of cyclodextrins on the solubility and antimycotic activity of sertaconazole: experimental and computational studies. J. Pharm. Sci. 91: 2408-415.

Received : November 02, 2008;

Accepted : August 24, 2009 